\title{
Hiệu quả gia cố nền đất yếu bằng phương pháp đầm nén động
}

\section{Tạ Văn Phấn ${ }^{1}$}

${ }^{1}$ Trường Đại học Thủy lợi

\section{TỪ KHOÁ \\ Hiệu quả}

Gia cố nền đất

Yếu

Phương pháp

Đầm nén động

\section{KEYWORDS}

\section{Efficiency}

Ground reinforcement

Weak

Method

Dynamic compaction

\begin{abstract}
TÓM TẮT
Đầm nén động là một phương pháp được sử dụng để tăng mật độ của đất khi có một số hạn chế nhất định dưới bề mặt mà các phương pháp khác không phù hợp. Quá trình này liên quan đến việc thả một quả nặng nhiều lần xuống đất với những khoảng thời gian cách nhau đều đặn. Tác động của sự rơi tự do tạo ra các sóng ứng suất giúp làm đặc chắc đất. Những sóng mạnh này có thể xuyên qua tới $10 \mathrm{~m}$. Mức độ nén phụ thuộc vào trọng lượng của búa, độ cao rơi xuống và khoảng cách giữa các vị trí mà búa được thả. Hầu hết các loại đất có thể được cải thiện bằng đầm nén động. Tiến độ thi công nhanh, tiết kiệm chi phí, thích ứng tốt với các dự án quy mô lớn.
\end{abstract}

\begin{abstract}
This study evaluates the possibility of using fly ash, a by-product of coal thermal power plants, in the Dynamic compaction is a method that is used to increase the density of the soil when certain subsurface constraints make other methods inappropriate. The process involves dropping a heavy weight repeatedly on the ground at regularly spaced intervals. The impact of the free fall creates stress waves that help in the densification of the soil. These stress waves can penetrate up to $10 \mathrm{~m}$. The degree of compaction depends on the weight of the hammer, the height from which the hammer is dropped, and the spacing of the locations at which the hammer is dropped. Most soil types can be improved with dynamic compaction. Fast construction progress, cost-effective, well-adapted to large-scale projects.
\end{abstract}

\section{Giới thiệu chung}

Đầm nén động là một trong những phương pháp gia cố nền đất lâu đời nhất được biết đến, được người La Mã sử dụng trước năm 100 trước Công nguyên và ở Hoa Kỳ vào đầu những năm 1800. Tuy nhiên, kể từ cuối những năm 60 của thế kỷ trước, phương pháp này được phát triển bởi ông Louis Menard, nhóm Menard đã áp dụng phương pháp này để gia cố, cải tạo nền đất cho hàng nghìn địa điểm với các loại cấu trúc và điều kiện nền đất rất khác nhau như sân bay, bến cảng, kho chứa hàng nặng, tòa nhà, bãi chôn lấp,... Thời gian gần đây, phương pháp này cũng đã được áp dụng thành công cho các chất thải mỏ, bãi chôn lấp, đất dễ sụp đổ, các vị trí có hố sụt, gia cố nền cho các dự án lớn ...

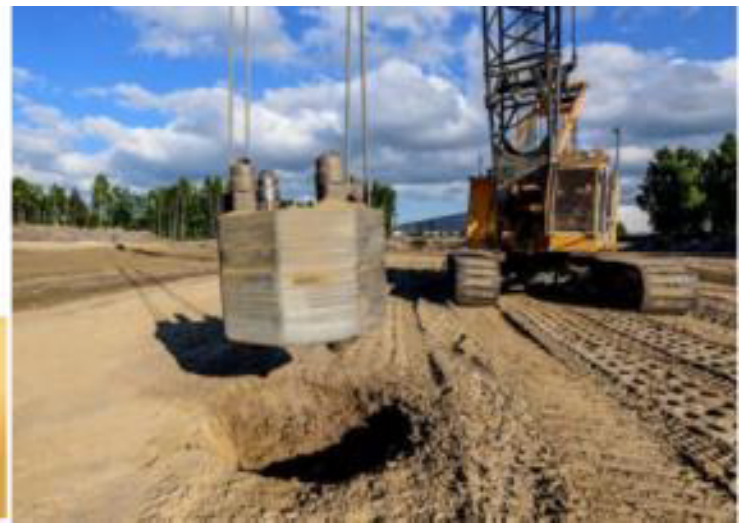

Hình 1. Phương pháp đầm nén động bằng quả nặng.

Đầm nén động là phương pháp làm chặt đất đạt tới một độ sâu tương đối lớn khi thực hiện đầm nén tại bề mặt đất. Đất được chặt lại do việc nâng và để rơi tự do của một quả chùy bằng thép nặng liên tục xuống mặt nền đất với những khoảng cách đều nhau. Trong lượng của quả chùy thường được sử dụng trong khoảng 6 tấn đến 30 tấn với chiều cao nâng chùy trong khoảng 9 đến $20 \mathrm{~m}$. Quá trình này thường được gọi bằng nhiều tên khác nhau như đầm nén cường độ cao, cố kết động, làm chặt sâu. 
Đầm nén động thực chất là một quá trình đầm đất, khi đó các hạt đất được đẩy lại gần nhau và sắp xếp lại trong quá trình đầm, các lỗ rỗng được giảm bớt làm đặc đất ở độ sâu đáng kể. Quá trình này xảy ra tương tự như khi có một dụng cụ được lăn và rung trên mặt đất. Tuy nhiên, việc đầm nén thông thường thường được sử dụng cho đất mới được đắp và đầm được sử dụng cho đất tự nhiên. Khác nhau nữa là đầm nén thường thì quả chùy được nâng lên rất thấp chỉ từ $20 \mathrm{~cm}$ đến $30 \mathrm{~cm}$. Độ ẩm của vật liệu được làm chặt thường được điều chỉnh trong khoảng độ ẩm tốt nhất chỉ một vài \% sai lệch. Thông thường đất được làm chặt nằm ở trên mực nước ngầm. Đối với đầm nén động, đất được làm chặt khi độ ẩm ở trạng thái tự nhiên. Độ sâu ảnh hưởng của đầm có thể đạt được đến lớp đất nằm dưới mực nước ngầm.

Các mục tiêu chính của đầm nén động là cải thiện các đặc tính về cường độ và khả năng chịu nén bằng cách tạo ra một khối vật liệu đông đặc đồng nhất hoặc bằng cách nén chặt tại các vị trí có tải trọng tập trung (ví dụ, tải trọng cột). Các đặc tính của đất được cải thiện làm tăng khả năng chịu lực và giảm độ lún, bao gồm cả độ lún khác nhau. Đầm nén động thường cho phép xây dựng các móng lan truyền thống bằng cách cung cấp khả năng chịu lực thường lên tới 100 - $150 \mathrm{kPa}$.

Bài báo này sẽ giới thiệu về hiệu quả của gia cố nền đất yếu bằng phương pháp đầm nén động.

\section{Phương pháp đầm nén động}

\subsection{Nguyên lý}

Nguyên lý cơ bản của kỹ thuật này là sự truyền sóng năng lượng cao qua một lớp đất có thể nén được để cải thiện các đặc tính địa kỹ thuật của nó ở độ sâu đáng kể.

\section{2. Ưu, nhược điểm}

\subsection{1. Ưu điểm}

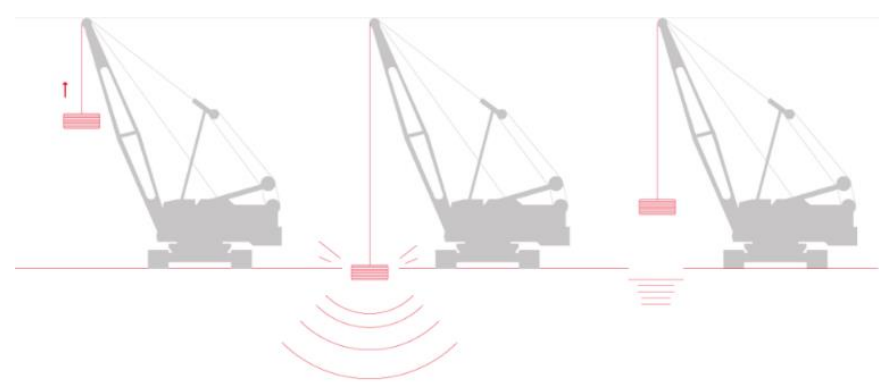

Hình 2. Nguyên lý phương pháp đầm nén động.

- Áp dụng cho nhiều loại đất có hàm lượng hạt mịn lên đến 20 \%, xuống độ sâu đáng kể lên đến 10 m, nhưng ảnh hưởng của nó đến độ sâu $12 \mathrm{~m}$. - Phương pháp này có hiệu quả đối với cả đất bão hòa và không bão hòa. Trong nền đất yếu, phương pháp đầm nén động đã được chứng minh là một giải pháp thay thế tốt hơn giải pháp gia tải nén trước, đóng cọc hay thay thế đất.

- Tiến độ thi công rất nhanh có thể đạt hơn 50000 m²/máy/ca/tháng. - Tiết kiệm chi phí.
- Thích ứng tốt với các dự án quy mô lớn lên đến vài triệu mét vuông, như các khu nhà công nghiệp, cơ sở dầu khí, công trình hạ tầng và các tòa nhà thương mại, đảo nhân tạo, kè đường bộ, đường sắt, cải tạo đất ... Tăng khả năng chịu lực của đất, giảm độ lún sau xây dựng và loại bỏ nguy cơ đất bị hóa lỏng trong trường hợp địa chấn.

- Có thể được thực hiện trước thiết kế nền móng.

- Kỹ thuật rất bền vững: không có chất thải, không dùng cốt liệu, không dùng xi măng, ...

\subsubsection{Nhược điểm}

- Phương pháp này không thể được sử dụng trong vòng 30 m cách các tòa nhà và $15 \mathrm{~m}$ từ các công trình ngầm.

- Đầm nén động là không thích hợp nếu độ sâu của nước ngầm nhỏ hơn $1,5 \mathrm{~m}$.

- Không thể được áp dụng nếu đất dính kết mềm nằm ở phần trên của đầm nén.

- Việc đầm nén động không hiệu quả khi đất có hàm lượng hạt mịn vượt quá $20 \%$.

- Yêu cầu cần có một chương trình thử nghiệm tại chỗ chuyên sâu (thử nghiệm xuyên tiêu chuẩn, thử nghiệm xuyên hình nón, thử nghiệm đồng hồ áp suất hoặc thử nghiệm độ lún tải, ...) để kiểm tra kết quả của quá trình nén chặt.

\subsection{Quá trình thi công}

Quả đầm được cẩu lên và thả xuống mặt đất nhiều lần. Các vị trí rơi thường nằm trên một mô hình lưới, khoảng cách giữa chúng được xác định bởi các điều kiện dưới bề mặt, tải trọng và hình dạng nền móng. Tác động năng lượng cao dẫn đến truyền sóng xung kích qua mặt đất đến độ sâu cần xử lý. Điều này làm giảm khoảng trống không khí và nước giữa các hạt đất dẫn đến đất được đặc chắc.

Chiều sâu ảnh hưởng (theo Robert Lucas):

$$
\mathrm{D}=\mathrm{n} .(\mathrm{W} \cdot \mathrm{H})^{1 / 2} \text {. }
$$

Trong đó: $\mathrm{D}$ : chiều sâu ảnh hưởng lớn nhất từ mặt đất (m),

W: trọng lượng quả tạ (tấn hay $\mathrm{kN}$ ),

H: chiều cao rơi tới mặt đầm (m),

n: hệ số lấy từ 0,4 - 0,8 tùy loại đất, trị số nhỏ nhất cho đất hạt mịn.

Độ sâu này cũng bị ảnh hưởng bởi các yếu tố khác như địa tầng của đất, mức độ bão hòa, cách thức rơi trọng lượng và sự hiện diện của bất kỳ lớp đất giảm chấn nào.

Số lượng rơi quả đầm tại mỗi vị trí có thể được tính toán bằng cách sử dụng công thức sau:

$$
\mathrm{E}=\text { (N.W.H.P)/S } \mathrm{S}^{2}
$$

Trong đó: E là năng lượng tác dụng,

$\mathrm{N}$ là số lần rơi tại mỗi vị trí điểm lưới,

W là trọng lượng thả,

H là độ cao thả,

$\mathrm{P}$ là số lần chuyền, 
S là khoảng cách lưới.

Mô hình lưới - khoảng cách các vết lõm được tính toán cụ thể. Sự sắp xếp của các điểm tác động và các thông số khác, thời gian nghỉ phụ thuộc vào đặc tính của đất được xử lý và vào kết quả thu được từ vùng thử nghiệm ban đầu.

Các lớp sâu hơn được nén chặt với khoảng cách rộng hơn và các lớp trên được nén chặt với khoảng cách hẹp hơn.

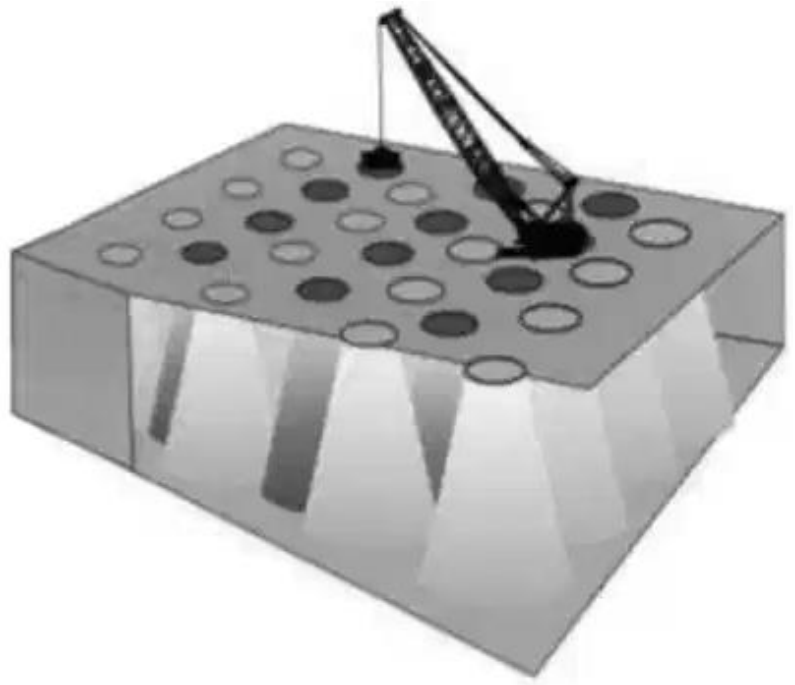

Hình 4. Khoảng cách nén chặt theo chiều sâu.

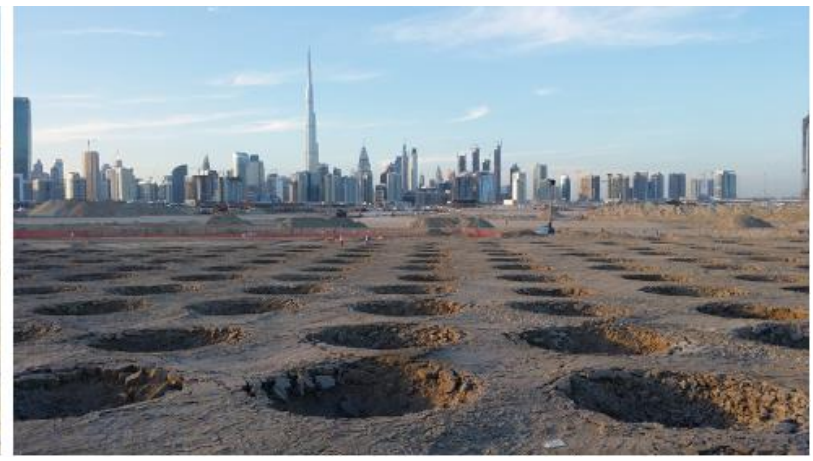

Hình 5. Khoảng cách các vết lõm thi công tại 1 dự án.

\section{Hiệu quả của phương pháp}

Phương pháp đầm nén động có thể áp dụng cho nhiều điều kiện đất khác nhau bao gồm cát rời bão hòa/không bão hòa, ngay cả với sự hiện diện của các túi bùn, cát cồn, đất lấp đầy vô cơ, đất cải tạo với các đặc tính và kích thước thay đổi hay các loại đất có kích thước lớn lẫn đá tảng, trầm tích bãi rác và đất dễ sụp đổ.

\section{Bảng 1.}

Hiệu quả đầm nén động với một số loại đất.

\begin{tabular}{|l|l|}
\hline \multicolumn{1}{|c|}{ Loại đất } & \multicolumn{1}{|c|}{ Hiệu quả } \\
\hline $\begin{array}{l}\text { Sỏi sạn và cát có }<10 \% \text { hạt bụi, không } \\
\text { phải là sét }\end{array}$ & Cực tốt \\
\hline $\begin{array}{l}\text { Cát có } 10-80 \% \text { hạt bụi và }<20 \% \text { hạt } \\
\text { sét }\end{array}$ & $\begin{array}{l}\text { Khá nếu khô, kém nếu } \\
\text { ướt }\end{array}$ \\
\hline Đất hạt mịn có hàm lượng mịn $>20 \%$ & Không dùng \\
\hline Đất đắp & Rất tốt \\
\hline
\end{tabular}

Phương pháp đầm nén động đã được sử dụng rộng rãi ở nhiều nước trên thế giới để nén chặt đất rời đến độ sâu lên đến $10 \mathrm{~m}$, nhằm tăng khả năng chịu lực, giảm độ lún sau xây dựng và giảm thiểu rủi ro hóa lỏng trong trường hợp địa chấn.

Công nghệ này ở Việt Nam còn chưa được phổ biến có lẽ vì xử lý nền đất yếu ở Việt Nam chưa tính đến hiện tượng hóa lỏng. Phương pháp này chủ yếu áp dụng cho xử lý nền cát rời rạc, có khả năng bị hóa lỏng cao khi có tác dụng của tải trọng rung động lớn như động đất. Một trong những dự án hiếm hoi có sử dụng phương pháp này ở nước ta là khu công nghiệp Fomosa - Hà Tĩnh đã xử lý nền đất yếu là khu vực có một lớp cát mịn dày khoảng $10 \mathrm{~m}$ (chỉ số SPT từ $3 \rightarrow 8$ ), dưới đó là 1 lớp sét yếu dày khoảng $5 \mathrm{~m}$ (có chỉ số $\mathrm{SPT}$ chỉ từ $1 \rightarrow 3$ ) với trọng lượng quả đầm khoảng 15 tấn, thả từ độ cao đến $20 \mathrm{~m}$ xuống. Dự án đã được hiệu quả về kinh tế và tiến độ thi công. 


\section{Kết luận}

Đầm nén động là một phương pháp được sử dụng để tăng mật độ của đất khi có một số hạn chế nhất định dưới bề mặt mà các phương pháp khác không phù hợp. Quá trình này liên quan đến việc thả một quả nặng nhiều lần xuống đất với những khoảng thời gian cách nhau đều đặn. Mức độ nén phụ thuộc vào trọng lượng của búa, độ cao rơi xuống và khoảng cách giữa các vị trí mà búa được thả. Hầu hết các loại đất có thể được cải thiện bằng đầm nén động.

Tiến độ thi công nhanh, tiết kiệm chi phí, thích ứng tốt với các dự án quy mô lớn.

\section{Tài liệu tham khảo}

[1]. Eun Chul Shin (2007), Gia cố đẩt bằng phương pháp đầm nén động, Tuyển tập bài giảng "Xử lý đất yếu và kỹ thuật nền móng công trình", Khoa Xây dựng và môi trường, ĐH Incheon, Hàn Quốc.

[2]. Tiêu chuẩn thiết kế công trình kháng chấn của ngành giao thông Hoa Kỳ (Design Standards for U.S. Transportation Infrastructure), trang 347 đến 349 -Thiết kế chống hóa lỏng theo phương pháp Dynamic compaction.

[3]. https://www.menard-group.com/soil-expert-portfolio/dynamiccompaction/

[4]. https://www.sciencedirect.com/topics/engineering/dynamic-compaction 